Hautarzt 2012 $\cdot 63: 448-451$

DOI 10.1007/s00105-012-2329-8

(c) Springer-Verlag 2012

E. Hölzle ${ }^{1}$ F.G. Bechara ${ }^{2}$

${ }^{1}$ Klinik für Dermatologie und Allergologie, Klinikum Oldenburg

${ }^{2}$ Klinik für Dermatologie, Venerologie und Allergologie, Ruhr-Universität Bochum

\title{
Pathophysiologie, Klinik und Diagnose der Hyperhidrose
}

drüsen aufgrund einer sympathischen und parasympathischen Dysfunktion [4].

Ekkrine Schweißdrüsen dienen in erster Linie der Thermoregulation. Unter physiologischen Bedingungen können die etwa 4 Mio. ekkrinen Schweißdrüsen der menschlichen Haut bis zu 21 Schweiß pro Stunde sezernieren. Besonders dicht sind die Schweißdrüsen an Handflächen, Fußsohlen und Achselhöhlen, in denen sich allein 20.000 bis 30.000 ekkrine Schweißdrüsen befinden [10]. Die Verdunstungskälte des Schweißes an der Hautoberfläche schützt den Körper vor Überwärmung. Im Bereich der Leistenhaut, an Handflächen und Fußsohlen dient das Schwitzen als Perspiratio insensibilis zur Anfeuchtung der Hornschicht und damit Optimierung der Reibung. Axilläres Schwitzen besitzt wahrscheinlich eine atavistische Funktion und ist Teil des axillären Duftorgans. Die durch bakterielle Zersetzung des apokrinen Schweißes präformierten geruchsaktiven Substanzen haften an der Hautoberfläche und werden durch die plötzlich einsetzende Befeuchtung durch den ekkrinen Schweiß mit dessen Verdunstung in die Umgebung freigesetzt. Thermoregulatorisches Schwitzen betrifft insbesondere den Körperstamm, wo Schweiß großflächig abdunsten kann. Schwitzen an Handflächen, Fußsohlen und in den Achselhöhlen wird überwiegend durch emotionale Stimuli getriggert. Diese sind geistige Anspannung, Schmerz, Angst und Befangenheit [2].

Vermehrtes Schwitzen im Sinne einer Hyperhidrose ist eine funktionelle Störung der Regelung der ekkrinen Schweiß-

\section{$\checkmark$ Eine Hyperhidrose liegt vor, wenn die Schweißabsonderung das zur Thermoregulation erforderliche Maß übersteigt oder der emotionalen Situation unangepasst ist.}

Die Hyperhidrose wird daher nicht anhand der objektiven Schweißmenge, sondern anhand der Fehlfunktion der Schweißdrüsen definiert [6].

\section{Klassifikation}

Die Hyperhidrose wird unterteilt in eine primäre essenzielle oder idiopathische Form und eine sekundäre somatisch bedingte oder symptomatische Form [6, 12]. Symptomatische Formen der Hyperhidrose treten auf bei endokrinologischen Erkrankungen, bei Zuständen mit einer Erhöhung der Katecholaminausscheidung, im Rahmen von Infektionserkrankungen und in Verbindung mit neurologischen Störungen. Eine kompensatorische Hyperhidrose entsteht, wenn durch neurologische Erkrankungen oder auch durch eine Miliaria die ekkrine Sekretion an größeren Hautarealen blockiert ist und dann zur Aufrechterhaltung der Thermoregulation die verbleibenden intakten Schweißdrüsen kompensatorisch überaktiviert werden. Eine physiologische Hyperhidrose tritt während der Akklimatisierung, in der Menopause oder als idiopathisches gustatorisches Schwitzen auf. Das Axonreflexschwitzen kann in der Umgebung von entzündlichen Hautveränderungen beobachtet werden. Der Naevus sudoriferus führt ebenfalls zu einer umschriebenen Hyperhidrose. Der primären essenziellen oder idiopathischen Form kann das vorwiegend emotional ausgelöste Schwitzen bei Hyperhidrosis axillaris, manuum oder pedum zugeordnet werden ( $\bullet$ Tab. 1).

Die meisten Formen der symptomatischen Hyperhidrose führen zu einem generalisierten Schwitzen, ähnlich dem thermoregulatorischen Muster. Neurologische Störungen führen häufig zu unterschiedlichen, teils segmentalen Verteilungsmustern und können dadurch Hinweise auf die Lokalisation und die Art der Erkrankung geben. Die idiopathische Hyperhidrose tritt als Hyperhidrosis axillaris, manuum oder pedum primär lokalisiert auf und wird daher häufig als fokale Hyperhidrose bezeichnet. Weitere typische Lokalisationen der fokalen Hyperhidrose sind Stirn und Capillitium. In besonders schweren Fällen kann ein Übergreifen auf die vordere und hintere Schweißrinne bis hin zum thermoregulatorischen Muster des Schwitzens vorliegen.

\section{Klinik der essenziellen Hyperhidrose}

\section{Epidemiologie}

Die Prävalenz der Hyperhidrose wird auf etwa 1 [1] bis 2,8\% [13] geschätzt. Sie ist am höchsten zwischen 18 und 65 Jahren. Männliche Patienten scheinen geringfü- 


\begin{tabular}{ll}
\hline Tab. 1 Pathogenese der Hyperhidrose. (Mod. nach [6, 9, 14]) \\
\hline Zugrunde liegende Störung & Beispiele \\
\hline Physiologische Hyperhidrose & Akklimatisierung, Klimakterium, gustatorisches Schwitzen \\
\hline Endokrinologische Störungen & Hyperpituitarismus \\
& Hyperthyreose \\
& Diabetes mellitus \\
\hline $\begin{array}{l}\text { Erhöhung der Katecholamin- } \\
\text { ausscheidung }\end{array}$ & Hypoglykämie \\
& Schock \\
& Phäochromozytom \\
\hline Neurologische Störungen & Halsrippe \\
& Karpaltunnelsyndrom \\
& Aurikulotemporales (Frey) Syndrom und andere Formen des \\
& nervalen gustatorischen Schwitzens \\
& Tabes dorsalis \\
& Syringomyelie \\
& Enzephalitis \\
& Diabetische Neuropathie \\
& Hemiplegie \\
& Plexuslähmung \\
& Grenzstrangläsion \\
\hline Kompensatorische Hyperhidro- & Ross-Syndrom \\
sis bei ausgedehnter Anhidrose & Diabetische Neuropathie \\
& Miliaria \\
& Grenzstrangläsion \\
\hline Axonreflexschwitzen & Entzündliche Hautläsionen \\
\hline Nävoide Fehlbildung & Naevus sudoriferus \\
\hline Idiopathische Hyperhidrose & Hyperhidrosis axillaris \\
& Hyperhidrosis manuum \\
& Hyperhidrosis pedum \\
& Hyperhidrosis facialis \\
& Generalisierte Hyperhidrose vom thermoregulatorischen Muster \\
\hline & \\
& \\
& \\
&
\end{tabular}

gig häufiger betroffen zu sein als Frauen. Die palmoplantare Hyperhidrosis beginnt oftmals bereits in der frühen Kindheit, axilläres Schwitzen setzt erst mit Beginn der Pubertät ein. Bei etwa 50\% der Hyperhidrotiker sind Handflächen oder Fußsohlen betroffen.

In bis zu $65 \%$ aller Fälle wird über eine familiäre Häufung berichtet. Bei der Hyperhidrosis palmaris wird ein autosomal-dominantes Vererbungsmuster mit Lokalisation des verantwortlichen Gens auf Chromosom 14 vermutet [5]. Durch eine unvollständige Penetranz kommt es nicht in allen Fällen zu einer Manifestation der Erkrankung.

\section{Manifestationsfaktoren und Schweregrad}

Bei axillärer und palmoplantarer Hyperhidrose wird das Schwitzen vorwiegend durch emotionale Stimuli ausgelöst. Die Hyperhidrose tritt meist anfallsartig und ausgelöst durch Stresssituationen auf, wobei die Reizschwelle so niedrig sein kann,
Hyperhidrose und darüber hinaus einer schweren Hyperhidrose entsprechen. In letzterem Fall rinnt Schweiß von der Achselhöhe und kann mehrere Schichten von Textilien durchnässen.

Die Handflächen und Fußsohlen von Hyperhidrotikern sind meist diffus gerötet, und abhängig von der Intensität des Schwitzens ist die Hornschicht mazeriert. Meist fühlen sich die Extremitäten infolge der erhöhten Verdunstungskälte kühl an. Lediglich feuchte Handflächen oder Fußsohlen würden einem leichten Schweregrad zugeordnet werden. Sind Schweißperlen sichtbar, so entspricht das einer mäßiggradigen Hyperhidrose, wobei dann auch zunehmend die dorsalen Seiten der Finger und Zehen von der Hyperhidrose mit betroffen sind. Bei einer stark ausgeprägten palmoplantaren Hyperhidrose tropft Schweiß von Handflächen oder Fußsohlen, und insbesondere das Schuhwerk wird durchfeuchtet $[7,8]$.

Neben diesen subjektiven Methoden zur semiquantitativen Einschätzung des Ausprägungsgrades der Hyperhidrose stehen kolorimetrische, hygrometrische und gravimetrische Verfahren zur Messung der Schweißdrüsensekretion zur Verfügung. Eine einfache quantitative Methode ist die Gravimetrie. Hierbei wird zuvor gewogenes Filterpapier über eine definierte Zeit, meist $1 \mathrm{~min}$, auf das zu erfassende Areal gelegt und dabei der sezernierte Schweiß von dem Papier aufgenommen und anschließend als Gewichtszunahme bestimmt. Als Grenzwerte für die Hyperhidrose werden an den Handflächen $20-30 \mathrm{mg} / \mathrm{min}$ und in den Achselhöhlen $50 \mathrm{mg} / \mathrm{min}$ angesehen [12].

Eine kolorimetrische Methode ist der Jod-Stärke-Test nach Minor. Hierbei wird eine 1,5\%ige Jodtinktur auf die zu untersuchende Hautstelle gepinselt und nach dem Antrocknen Getreidestärke dünn darüber gestreut und mittels eines Wattebausches fein verteilt. Sobald Schweiß hinzutritt, entsteht ein Farbumschlag nach dunkelbraun bis violett durch die Bildung eines gefärbten Jod-Stärke-Komplexes. Die Intensität und Ausdehnung des Farbumschlages ermöglicht eine sehr gute semiquantitative Abschätzung der Stärke des Schwitzens.

Hygrometrische Messungen registrieren die Wasserabgabe der Haut, bezogen 


\section{Tab. 2 Diagnostische Kriterien für eine primäre fokale Hyperhidrose. (Mod. [9, 14])}

\begin{tabular}{|c|c|}
\hline Hauptkriterium & $\begin{array}{l}\text { Fokales sichtbares Schwitzen, das über ein normales Maß hinausgeht und } \\
\text { seit wenigstens } 6 \text { Monaten besteht }\end{array}$ \\
\hline Nebenkriterien & $\begin{array}{l}\text { 1) Primärer Befall von Achselhöhlen, Handflächen, Fußsohlen oder } \\
\text { Stirn-Kopf-Bereich } \\
\text { 2) Bilaterale und symmetrische Ausprägung } \\
\text { 3) Wenigstens } 1 \text { Episode pro Woche } \\
\text { 4) Kein Schwitzen während des Nachtschlafes } \\
\text { 5) Erstmanifestation vor dem 25. Lebensjahr } \\
\text { 6) Positive Familienanamnese } \\
\text { 7) Beeinträchtigung im Alltag }\end{array}$ \\
\hline
\end{tabular}

Die Diagnose ist mit einer Sensitivität von 99\% und einer Spezifität von $80 \%$ zu stellen, wenn das Hauptkriterium und wenigstens 4 der 7 Nebenkriterien erfüllt sind.

Tab. 3 Stufenschema zur Behandlung der primären fokalen Hyperhidrose

\begin{tabular}{|c|c|c|}
\hline Stufe & Axilläre Hyperhidrose & Palmoplantare Hyperhidrose \\
\hline \multirow[t]{2}{*}{ 1. Stufe } & A: Kommerzielle Antiperspiranzien & A: Kommerzielle Antiperspiranzien \\
\hline & $\begin{array}{l}\mathrm{B}: 15-20 \% \mathrm{AlCl}_{3} 6 \mathrm{H}_{2} \mathrm{O} \text { über Nacht } \\
\text { (richtige Anwendung zur Vermeidung } \\
\text { von Hautreizungen) }\end{array}$ & $\begin{array}{l}\mathrm{B}: 20-30 \% \mathrm{AlCl}_{3} 6 \mathrm{H}_{2} \mathrm{O} \text { über Nacht mög- } \\
\text { lichst unter Okklusion (richtige Anwen- } \\
\text { dung zur Vermeidung von Hautreizungen) }\end{array}$ \\
\hline 2. Stufe & $\begin{array}{l}\text { Leitungswasseriontophorese mit } \\
\text { speziellen Elektroden }\end{array}$ & Leitungswasseriontophorese \\
\hline 3. Stufe & $\begin{array}{l}\text { Intradermale Injektion von Botulinum- } \\
\text { toxin A }\end{array}$ & $\begin{array}{l}\text { Intradermale Injektion von Botulinum- } \\
\text { toxin } A^{a}\end{array}$ \\
\hline 4. Stufe & $\begin{array}{l}\text { A: Lokale Schweißdrüsenentfernung } \\
\text { B: Endoskopische thorakale Sympath- } \\
\text { ektomie }^{\text {b }}\end{array}$ & 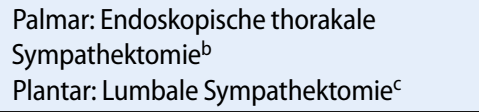 \\
\hline \multicolumn{3}{|c|}{$\begin{array}{l}\text { aPalmoplantare Injektionen erfordern wegen der Schmerzhaftigkeit meist zusätzliche leitungsanästhetische } \\
\text { Verfahren. 'Die Patienten müssen über die Wirksamkeit und mögliche Komplikationen, einschließlich einer } \\
\text { kompensatorischen Hyperhidrose, aufgeklärt werden. 'Die Behandlung ist häufig mit Nebenwirkungen belastet, } \\
\text { daher ist strengste Indikationsstellung erforderlich. }\end{array}$} \\
\hline
\end{tabular}

auf die Messfläche. Gemessen wird allerdings nur die verdunstende Feuchtigkeit, sodass diese Geräte insbesondere zur Bestimmung des transepidermalen Wasserverlustes und der Perspiratio insensibilis geeignet sind. Kumuliert Flüssigkeit auf der Hautoberfläche, so wird diese messtechnisch nicht mit erfasst.

Einschränkend für alle Testverfahren muss angemerkt werden, dass sie nur eine Momentaufnahme wiedergeben und nicht immer dem oft situativen Charakter fokaler Hyperhidrosen gerecht werden.

\section{Lebensqualität und Krankheitswert}

Die Hyperhidrose kann zu einer schwerwiegenden Einschränkung der Lebensqualität bei betroffenen Patienten führen. Sie wird durch soziale, berufliche und medizinische Probleme bedingt [14]. Das profuse Schwitzen schädigt Kleidung, Schuhe und empfindliche Materialien, die durch schweißnasse Hände befeuch- tet werden. Sichtbare Achselnässe und ein kühler, feuchter Händedruck wirken sozial abstoßend. Die Patienten sind gehemmt im Beruf, in ihrer sozialen Interaktion und auch bei Sport und anderen Freizeitbeschäftigungen. Das ausgeprägte Schwitzen oder auch ein unangepasstes mäßiges Schwitzen bedeutet emotionalen und psychologischen Stress und verstärkt wiederum Verunsicherung und Stress und führt schließlich zu einem Circulus vitiosus. Der negative Effekt einer primären Hyperhidrose auf die Lebensqualität wurde mithilfe von Fragebögen an einer größeren Patientengruppe überprüft, und er übertraf zum Teil die Einschränkungen aufgrund zahlreicher anderer chronischer Erkrankungen [11].

Neben den psychosozialen Auswirkungen kann die Hyperhidrosis jedoch auch zu ernsthaften Gesundheitsstörungen Anlass geben. Die Mazeration der Haut durch ständige Feuchtigkeit begünstigt bakterielles und mykotisches Wachstum und bildet die Grundlage der Intertrigo.
An den Füßen ist das verstärkte bakterielle Wachstum die Ursache der Bromhidrose und des Keratoma sulcatum. Auch eine Tinea pedum ist häufig mit vermehrtem Schwitzen verbunden. Als schwere Komplikation ist hierbei der mazerative Fußinfekt durch das Überwuchern gramnegativer Keime gefürchtet.

\section{Diagnostik}

Wichtig ist die Abgrenzung sekundärer symptomatischer Formen der Hyperhidrose von der primären genuinen Form. In der klinischen Alltagssituation stehen meist keine objektiven Daten zur Bewertung der sezernierten Schweißmenge zur Verfügung. Es hat sich daher bewährt, eine primäre fokale Hyperhidrose anhand der Anamnese und der klinischen Ausprägung zu diagnostizieren ( $\bullet$ Tab. 2).

Ist die Diagnose einer idiopathischen fokalen Hyperhidrose aufgrund der oben genannten Kriterien gesichert, so sind keine weiteren laborchemischen oder bildgebenden Untersuchungen indiziert. Besteht jedoch der Verdacht auf eine sekundäre symptomatische Hyperhidrose, so muss eine symptomorientierte Untersuchung bis zu einer klaren diagnostischen $\mathrm{Ab}$ grenzung erfolgen, sodass möglichst eine kausale Therapie erfolgen kann.

\section{Therapieoptionen}

Die Behandlung der idiopathischen Hyperhidrose folgt einem Stufenschema (- Tab.3). Es umfasst allgemeine Maßnahmen, die Anwendung von Lokaltherapeutika, die physikalische Behandlung mit Leitungswasseriontophorese, die Gabe von Systemtherapeutika, die Injektion von Botulinumtoxin A und schließlich operative Behandlungsmodalitäten.

Allgemeine Behandlungsmaßnahmen, die immer unterstützend ergriffen werden sollten, umfassen das Tragen luftdurchlässiger Kleidung, möglichst aus feuchtigkeitsabsorbierendem Gewebe, sowie Schuhe aus Leder, die eine Durchlüftung ermöglichen. Weitere Maßnahmen sind eine Gewichtsreduktion, falls erforderlich, sowie die Einschränkung von übermäßigem Genuss von scharfen Gewürzen und Koffein oder Nikotin. Entspannungstraining oder psychosomatische Betreuung 
sind geeignet, psychologischen Faktoren entgegenzuwirken und Stress abzubauen.

Die weiteren genannten differenten Behandlungsmodalitäten werden - entsprechend dem Schweregrad und der Lokalisation der primären fokalen Hyperhidrose - stufenförmig eingesetzt. Einer nationalen Leitlinie (AWMF) und internationalen Empfehlungen [14] folgend, ergibt sich der in $\bullet$ Tab. 3 dargestellte Therapiealgorithmus.

Sowohl bei der axillären wie auch bei der palmoplantaren Hyperhidrose in moderater Ausprägung können primär kommerzielle Antiperspiranzien auf der Basis von Aluminiumsalzen versucht werden. Bei mäßiggradiger, insbesondere schwer ausgeprägter Hyperhidrose empfehlen sich Lösungen von Aluminiumchlorid-Hexahydrat axillär in einer Konzentration von 15-20\% und palmoplantar in einer Konzentration von $20-30 \%$.

Die zweite Stufe der Behandlung der palmoplantaren Hyperhidrose ist die Leitungswasseriontophorese, die allgemein als Standardtherapie bei dieser Indikation gilt. Für die Behandlung der axillären Hyperhidrose mit Leitungswasseriontophorese sind spezielle Elektroden in Form von angefeuchteten Schaumstoffpolstern erforderlich. Die Datenlage ist hierbei jedoch im Vergleich zur palmoplantaren Anwendung weniger gesichert.

Die dritte Stufe der Therapie umfasst die Injektionen von Botulinumtoxin A. Diese sind in der Achselhöhle leicht durchzuführen, an den Handflächen und an den Fußsohlen ist die Anwendung durch die Schmerzhaftigkeit der Injektionen eingeschränkt, und die Behandlung erfordert anästhesierende Maßnahmen.

Die vierte Stufe umfasst operative Maßnahmen, die in der Achselhöhe durch eine lokale Schweißdrüsenentfernung mittels Kürettage, Saugkürettage oder auch Exzision der Schweißdrüsenfelder erreicht werden kann. Ein Goldstandard chirurgischer Eingriffe axillär ist bislang nicht definiert. Die endoskopische thorakale Sympathektomie kann als Ultima Ratio bei der palmaren Hyperhidrosis und in Ausnahmefällen auch bei störendem Schwitzen im Kopfbereich eingesetzt werden. Von einer lumbalen Sympathektomie zur Behandlung der Hyperhidrose der Fußsohlen wird meist abgeraten.
Die nachfolgenden Beiträge fokussieren jeweils auf eine Behandlungsmodalität der Hyperhidrose, sodass insgesamt eine detaillierte Darstellung der aktuellen therapeutischen Möglichkeiten gegeben wird. Bezüglich der operativen Therapieverfahren wird auf den rezenten Artikel von Bechara [3] verwiesen.

\section{Korrespondenzadressen}

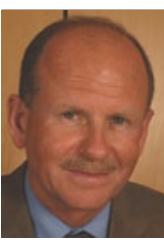

Prof. Dr. E. Hölzle

Klinik für Dermatologie und

Allergologie,

Klinikum Oldenburg

Rahel-Straus-Str. 10,

26133 Oldenburg

hoelzle.erhard@klinikum-

oldenburg.de

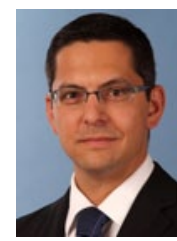

PD Dr. F.G. Bechara

Klinik für Dermatologie, Venerologie und Allergologie, Ruhr-Universität Bochum Gudrunstr. 56, 44791 Bochum f.bechara@klinikum-bochum. de

Interessenkonflikt. Die korrespondierenden Autoren geben an, dass kein Interessenkonflikt besteht.

\section{Literatur}

1. Adar R, Kurchin A, Zweig A, Mozes M (1977) Palmar hyperhidrosis and its surgical treatment: a report of 100 cases. Ann Surg 186:34-41

2. Allen JA, Armstrong JE, Roddie IC (1973) The regional distribution of emotional sweating in man. J Physiol 235:749-759

3. Bechara FG (2009) Aktuelle Therapie der fokalen Hyperhidrose. Operative Verfahren. Hautarzt 60:538-545

4. Chu EC, Chu NS (1997) Patterns of sympathetic skin response in palmar hyperhidrosis. Clin Auton Res 7:1-4

5. Higashimoto I, Yoshiura K, Hirakawa N et al (2006) Primary plamar hyperhidrosis locus maps to 14q11.2q13. Am J Med Genet A 140:567-572

6. Hölzle E (1983) Physiopathologic aspects and clinical pictures in hyperhidrosis. Hautarzt 34:596-604

7. Hölzle E (2000) Antiperspirants. In Gabard B, Lesner P, Surber C, Treffel P(Hrsg): Dermato-pharmacology of topical preparations. Springer, Berlin, S 401-416

8. Hölzle E (2002) Pathophysiology of sweating. In: Kreyden et al (Hrsg) Hyperhidrosis and botulinum toxin in dermatology. Karger, Basel

9. Hornberger J, Grimes K, Naumann M et al (2004) Recognition, diagnosis, and treatment of primary focal hyperhidrosis. J Am Acad Dermatol 51:274286

10. Kuno Y (1956) Human perspiration. Charles C Thomas, Springfield, USA
11. Naumann MK, Hamm H, Lowe NJ (2002) Effect of botulinum toxin type $A$ on quality of life measures in patients with excessive axillary sweating: a randomized controlled trial. Br J Dermatol 147:12181226

12. Sonntag M, Ruzicka T (2005) Hyperhidrose - Ursachen und aktuelle Behandlungsmöglichkeiten. Psychoneuro 31:315-320

13. Strutton DR, Kowalski JW, Glaser DA, Stang PE (2004) US prevalence of hyperhidrosis and impact on individuals with axillary hyperhidrosis: results from a national survey. Am Acad Dermatol 51:241-248

14. Walling HW (2011) Clinical differentiation of primary from secondary hyperhidrosis. J Am Acad Dermatol 64:690-695

15. Weber A, Heger S, Sinkgraven R et al (2005) Psychosocial aspects of patients with focal hyperhidrosis. Marked reduction of social phobia, anxiety and depression and increased quality of life after treatment with botulinum toxin $\mathrm{A}$. Br J Dermatol 152:342-345 